\title{
Eficiência informacional em anúncios de criação de joint-ventures no mercado acionário brasileiro ${ }^{1}$
}

\section{Informational efficiency in ads for joint ventures creation in the Brazilian stock market}

\author{
Ivan Fernandes da Cruz \\ Gabriel Augusto de Carvalho \\ Felipe Dias Paiva \\ Lívia Maria de Pádua Ribeiro \\ Uajará Pessoa Araújo \\ Mestre em Administração. Centro Federal de Educação Tecnológica de Minas Gerais (CEFET-MG) - Brasil. \\ ivanfernandes26@bol.com.br \\ Mestre em Administração. Centro Federal de Educação Tecnológica de Minas Gerais (CEFET-MG) - Brasil. \\ ga09carvalho@gmail.com \\ Doutor em Administração. Centro Federal de Educação Tecnológica de Minas Gerais (CEFET/MG) - Brasil. \\ fpaiva@dcsa.cefetmg.br \\ Doutora em Administração. Centro Federal de Educação Tecnológica de Minas Gerais (CEFET/MG) - Brasil. \\ livia.padua2014@gmail.com \\ Doutor em Administração. Centro Federal de Educação Tecnológica de Minas Gerais (CEFET/MG) - Brasil. \\ uajara@dcsa.cefetmg.br
}

\section{RESUMO}

Este estudo teve como objetivo verificar a presença da eficiência semiforte, no mercado brasileiro de ações, quando do anúncio da criação de joint-ventures. O período analisado no estudo foi de 24/05/2012 a 24/05/2017, sendo obtida uma amostra de 12 anúncios de criação de novas joint-ventures, nas quais pelo menos um dos acionistas foi uma empresa brasileira de capital aberto. Posteriormente, foi empregada a metodologia de estudo de eventos. Foram estimados os retornos esperados por meio do modelo de mercado, e então calculados os retornos anormais (AR) e os retornos anormais acumulados (CAR) durante a janela de evento. Por fim, foram realizados testes estatísticos para a média dos retornos anormais, a fim de verificar se os valores foram significativos. Os resultados do trabalho evidenciaram uma forte tendência de queda nos ARs ao longo da janela de evento, consequentemente o CAR foi fortemente negativo, atingindo um valor de $-4,17 \%$ ao final da janela de evento. Tal fato indica que a criação de uma joint-venture, foi vista como negativa pelo mercado. Sobre a significância estatística dos resultados, ressalta-se que, apenas nos dois dias que antecederam o anúncio da criação da joint-venture o AR foi significativo, nos demais dias o mercado se comportou de maneira eficiente, com os AR não sendo estatisticamente diferente de zero. $\mathrm{O}$ resultado desta pesquisa é relevante para a decisão dos gestores que avaliam a possibilidade de estabelecer uma nova jointventure, mostrando os impactos que essa decisão pode ocasionar nas ações de sua empresa.

Palavras-chave: Eficiência de Mercado. Eficiência Semiforte. Estudo de Evento. Joint-Venture.

\begin{abstract}
This study aimed to verify the presence of semi-strong efficiency in the Brazilian stock market when the creation of joint ventures was announced. The period analyzed in the study was from May 24, 2012 to May 24, 2017, with a sample of 12 announcements about the creation of new joint ventures, in which at least one of the shareholders was a publicly traded Brazilian company. Subsequently, the event study methodology was employed. Expected returns were estimated using the market model, and then abnormal returns (AR) and cumulative abnormal return (CAR) were calculated during the event window. Finally, statistical tests were performed for the average of the abnormal returns in order to verify if the values were significant. The results of the work showed a strong downward trend in the ARs throughout the event window, consequently the CAR was strongly negative, reaching a value of $-4.17 \%$ at the end of the event window. This fact indicates that the creation of a joint venture was seen as negative by the market. Regarding the statistical significance of the results, it is noteworthy that, only in the two days prior to the announcement of the creation of the joint venture, the AR was significant, in the other days the market behaved efficiently, with the AR not being statistically different from zero. The result of this research is relevant to the decision of managers who evaluate the possibility of establishing a new joint venture, showing the impacts that this decision may have on their company's stocks.
\end{abstract}

Keywords: Market Efficiency. Semi-Strong Efficiency. Event Study. Joint Venture.

Recebido em 23/09/2019. Aprovado em 23/10/2019. Avaliado pelo sistema double blind peer review. Publicado conforme normas da APA. http://dx.doi.org/10.22279/navus.2020.v10.p01-16.1080

\footnotetext{
${ }^{1}$ Trabalho publicado em versão preliminar nos anais do XX SEMEAD - ISSN 2177-3866 com reajustes para a revista.
} 


\section{INTRODUÇÃO}

A Hipótese de Eficiência de Mercado (HEM), desenvolvida por Fama (1970), constitui-se como um dos pilares da teoria moderna de finanças. A HEM dedica-se ao estudo e análise do modo como as informações estão disponíveis para os investidores e em como elas são incorporadas aos preços das ações. Dados os diferentes níveis de informação, Fama (1970) propõe três formas de eficiência: eficiência fraca, semiforte e forte, sendo que, a medida em que mais informações são precificadas pelo mercado, maior é o seu nível de eficiência.

Em um mercado considerado eficiente os agentes têm acesso à todas as informações sem custos relevantes, e como consequência disso, novas informações são imediatamente incorporadas aos preços, o que inviabiliza estratégias de negociação que visem obter retornos anormais ao explorar essas informações. Conforme apontado por Camargos e Barbosa (2015), um mercado com essas características tende a crescer e a impulsionar o desenvolvimento econômico do país.

Com relação à eficiência semiforte, Fama (1970) destaca que em um mercado assim definido os preços dos ativos incorporam imediatamente todas as informações públicas disponíveis. O método do estudo de evento é descrito por Fama (1991) como uma alternativa para verificar a presença da eficiência semiforte em um mercado. Nessa forma de eficiência, conforme destacam Fé Júnior, Nakao e Ribeiro (2015), nenhum investidor é capaz de obter retornos anormais ao manter uma estratégia de negociação a partir de novas informações públicas, pois essas são rapidamente incorporadas aos preços dos ativos, sendo a velocidade e a precisão com que essa informação é absorvida pelos preços um indicativo do nível de eficiência do mercado.

Um fato relevante é uma informação pública, que impacta a expectativa de retorno que os investidores têm com a empresa, devendo ser rapidamente precificado em um mercado eficiente na forma semiforte. Os fatos relevantes podem ser definidos de maneira sucinta, de acordo com a Instrução 358 de Janeiro de 2002 da Comissão de Valores Mobiliários (CVM), como sendo qualquer ato ou fato relacionado aos negócios que impactam na cotação das ações da empresa, nas decisões de comprar, vender ou manter um ativo de um investidor ou na decisão de um acionista exercer direitos cabíveis por sua condição.

Em vista disso, a criação de uma joint-venture configura-se como um fato relevante, por impactar no lucro esperado e no posicionamento estratégico das empresas acionistas e, consequentemente, nas decisões dos investidores. De acordo com a American Bar Association (2006), uma joint-venture é uma relação estabelecida entre duas ou mais empresas com vistas a atingir um objetivo particular, nessa relação normalmente é criada uma nova empresa, que tem suas ações compartilhadas entre as empresas que se relacionaram previamente.

Esse tipo de relação, conforme apontado por Johnson e Houston (2000), gera ganhos para as empresas envolvidas de alguma forma, sejam em decorrência da sinergia, pela redução dos custos de transação, ou ainda por algum outro fator. Com isso, espera-se que o anúncio da criação de uma joint-venture gere impactos nas ações das empresas acionistas. Contudo, esse fato é imediatamente precificado pelos investidores em um mercado com eficiência semiforte.

Essa pesquisa justifica-se por analisar o efeito observado no comportamento das ações de empresas brasileiras envolvidas no processo de criação de joint-ventures, sendo destacados aspectos da (in)eficiência dos mercados de capitais para esse tipo de evento. Desse modo, os resultados da pesquisa são proveitosos para a tomada de decisão de investidores e empresas, e também para os reguladores de mercado, ao apontar o grau de eficiência do mercado em relação a esse tipo de anúncio.

Posto isto, o objetivo da pesquisa consiste em verificar a presença da eficiência semiforte, no mercado brasileiro de ações, para o evento da divulgação da criação de uma joint-venture. Para tanto, o período analisado foi de 24/05/2012 a 24/05/2017, no qual foram obtidos 12 anúncios do estabelecimento desse tipo de relação no período, e então foi empregada a metodologia de estudo de eventos.

O presente artigo está organizado da seguinte forma, após essa introdução é apresentado o referencial teórico a respeito dos estudos que versam sobre a eficiência de mercado, com ênfase nos estudos de eventos, e também sobre a importância e os impactos das joint-ventures para as empresas envolvidas em sua criação. Posteriormente são descritos os procedimentos metodológicos adotados para o cumprimento do 
objetivo do estudo, na continuação é desenvolvida uma análise dos resultados e na última seção são apresentadas algumas conclusões e considerações inferidas a partir do desenvolvimento dessa pesquisa.

\section{REFERENCIAL TEÓRICO}

Dado o objetivo deste estudo, o referencial teórico está estruturado em três subseções. A primeira discorre sobre a evolução do conceito de eficiência de mercado e suas três formas. A segunda subseção é dedicada à análise da eficiência semiforte e os estudos de eventos, apresentando um panorama geral deste tipo de estudo. Por fim, são tratados os aspectos e os impactos esperados pela criação de uma joint-venture.

\subsection{Eficiência de mercado}

O comportamento dos preços das ações e a oportunidade de ganhos anormais estão associados ao nível de eficiência do mercado no qual as negociações ocorrem, sendo assim, torna-se matéria relevante de estudos as teorias sobre esse tema. Em artigo seminal sobre a HEM, Fama (1970) define um mercado eficiente como sendo aquele em que todas as informações disponíveis estão refletidas nos preços dos ativos. Camargos e Barbosa (2015) ressaltam que a intensidade dos efeitos das informações nos preços dos ativos é afetada pelo contexto do mercado, pela relevância da informação e, principalmente, pela capacidade de interpretação e absorção das novas informações pelos investidores.

Fama (1970) ressalta que um mercado eficiente cumpre um papel importante ao fornecer sinais corretos para a tomada de decisão dos investidores ao alocar seus recursos. Em estudo mais recente, Gabriel, Ribeiro e Ribeiro (2013) salientam que essa teoria considera o homem como um ser racional, que analisa todas as informações disponíveis e sempre toma a melhor decisão visando a maximização do seu retorno.

Fama (1970) define três condições suficientes para que um mercado seja considerado eficiente, a saber: (i) ausência de custos de transação nas negociações dos títulos; (ii) qualquer participante do mercado acessa as informações disponíveis sem nenhum custo; (iii) expectativas homogêneas dos investidores em relação aos efeitos das informações nos preços dos títulos. Essas condições são consideradas suficientes, mas não necessárias para que um mercado seja eficiente, em determinadas circunstâncias a falta de uma dessas condições pode ser absorvida pelo mercado sem culminar em ineficiência, o que é apontado por Fama (1970) como uma constante nos mercados reais.

Desse modo, Fama (1970) aponta que essa é uma definição ampla e não empiricamente testável, sendo necessário um modelo que estime quais seriam os retornos esperados para o ativo em condições de equilíbrio, para que então esses valores sejam comparados aos verificados e assim atestada a (in)eficiência do mercado. Com isso, na definição de Fama (1970) se o mercado se comportar de maneira eficiente, a diferença entre esses dois valores será estatisticamente igual a zero, sendo inviabilizadas estratégias de negociação baseadas em informações.

Camargos e Barbosa (2003) apontam que, em relação ao nível informacional, o mercado de capitais pode apresentar diferentes graus de desenvolvimento, relacionados a velocidade e a acurácia com que os preços incorporam as informações. Dadas as diferentes condições como as informações estão disponíveis e são incorporadas nos preços dos ativos, Fama $(1970 ; 1991)$ define três formas de eficiência informacional para um mercado.

A primeira delas consiste na forma fraca de eficiência de mercado, nessa, conforme descreve Fama (1970), os preços presentes devem refletir toda a informação contida nos preços passados. Um mercado eficiente na forma fraca impossibilita qualquer estratégia fundamentada no histórico de preços, um exemplo disso seria a inviabilização da análise técnica como estratégia de negociação. Sobre a eficiência fraca de mercado, cabe ainda ressaltar que, em Fama (1991) os estudos dessa forma são renomeados como testes de previsibilidade dos retornos, sendo também sua definição ampliada, com a incorporação de novas variáveis estruturais nos testes.

A segunda forma consiste na eficiência semiforte de mercado, sendo essa definida por Fama (1970) a partir dos testes que são feitos para se verificar a velocidade de ajuste dos preços dos ativos a novas 
informações públicas. Em um mercado considerado eficiente nessa forma, os preços dos ativos incorporam imediatamente todas as informações públicas disponíveis que os impactam. Os testes da eficiência semiforte de mercado são definidos por Fama (1991) como estudos de eventos.

Por fim, a terceira forma de eficiência de mercado é a eficiência forte, na qual, de acordo com Fama (1970), toda a informação disponível está incorporada nos preços dos ativos, com isso nem mesmo um insider - agente que possui acesso a informações privadas - é capaz de obter um retorno acima do esperado. Fama (1991) nomeia os testes para a eficiência forte de mercado como testes de informação privada. Camargos e Barbosa (2003) destacam que os trabalhos empíricos têm dado suporte apenas para as formas fraca e semiforte de eficiência de mercado.

Ainda sobre os níveis de eficiência de mercado, cabe destacar a colocação de Maluf Filho (1991), de que tais formas seguem um critério de dominância, no qual o nível semiforte exige que sejam cumpridos os pressupostos do nível fraco, e o nível forte demanda que previamente estejam satisfeitas as condições dos níveis fraco e semiforte de eficiência. Como consequência das ideias acima salientadas, a HEM implica que as negociações de ações são efetivadas a um preço justo, incorporando o fluxo de caixa esperado, dadas as informações disponíveis. Fama (1991) ressalta, em relação ao comportamento dos preços, que eles seguem a chamada random walk, ou passeio aleatório, o que faz com que as variações dos preços sejam imprevisíveis. Conforme destacado por Perobelli e Ness Junior (2000), a random walk não determina um efeito nulo das informações passadas sobre a distribuição dos retornos, mas sim que a ordem dos retornos passados não influi sobre os retornos futuros.

Em resumo, Gabriel et al. (2013) ressaltam que a HEM considera que as informações relevantes são incorporadas imediatamente aos preços das ações, o que elimina a possibilidade de retornos anormais. Desse modo, dada a proposta desse trabalho de observar os efeitos gerados pelo anúncio da criação de uma jointventure nos preços das ações das empresas envolvidas, seu caráter consiste então em analisar a presença da eficiência semiforte de mercado neste contexto, por meio de um estudo de evento.

\subsection{Estudo de evento}

Tal como descrito por Bergmann, Savoia, Souza e Mariz (2015), um estudo de evento tem como objetivo de observar como um evento específico impacta o comportamento de uma segunda variável. No contexto da HEM, Fama (1970) salienta que um estudo de evento observa o comportamento dos preços das ações nas datas próximas ao dia da divulgação de uma nova informação pública, que afete os retornos esperados para o ativo.

French e Roll (1986) definem as informações públicas como sendo as informações que se tornam conhecidas em dado momento do tempo e, consequentemente, afetam os preços das ações, sendo um mercado eficiente quando os preços se ajustam antes que qualquer agente possa explora-la no mercado. Em conformidade com Fama (1991), quando há um anúncio de uma nova informação é possível demarcar sua data de ocorrência, para então observar como o preço do ativo reagiu nas datas próximas ao evento, e assim apontar o grau de eficiência do mercado em questão.

Bergmann et al. (2015) ressaltam que um estudo de evento deve analisar o comportamento dos preços antes e depois da divulgação de uma nova informação, para com isso observar a reação do mercado após o evento, e também se houve vazamento de informações, alterando o comportamento dos preços no período imediatamente anterior a divulgação do novo fato. Esses autores destacam ainda a importância de uma janela de estudo que não seja muito curta, de modo a não permitir o reconhecimento de possíveis vazamentos de informação, nem muito longa, para evitar que o comportamento dos dados esteja susceptível a outros eventos.

Damodaran (2001) aponta para a existência de três reações distintas do mercado na existência de uma nova informação relevante, são elas: (i) reação imediata e de forma adequada do mercado à nova informação, o que confirma a hipótese de eficiência semiforte; (ii) reação gradual do mercado, com um aumento progressivo dos preços, situação que gera a possibilidade de operações de arbitragem até o ajuste completo 
dos preços; (iii) reação imediata, mas inadequada, dos preços, com a correção sendo feita nos períodos seguintes.

Com isso, um estudo de evento parte da identificação de uma nova informação, a qual espera-se que impacte os preços dos ativos, e de sua respectiva data de ocorrência, para que então seja observado o comportamento dos preços em uma janela no entorno dessa data. Campbell, Lo e MacKinlay (1997) descrevem sete etapas necessárias para a realização de um estudo de evento, são elas: (i) definição do evento; (ii) definição dos critérios para amostra; (iii) seleção dos métodos para cálculo dos retornos normais e anormais; (iv) estimação dos retornos; (v) procedimento de testes dos retornos; (vi) resultados empíricos; e, (vii) interpretação e conclusão.

Esse tipo de estudo é empregado em diferentes contextos na pesquisa sobre a eficiência dos mercados de capitais, o que é possível de se atestar por meio de uma revisão da literatura recente sobre finanças. Desse modo, na sequência são apresentadas algumas evidências empíricas sobre a forma de eficiência semiforte, com especial atenção para estudos realizados no contexto do mercado brasileiro ou em situações de fusões e aquisições e anúncio da criação de joint-ventures, situações similares ao evento em análise nesse estudo.

\subsubsection{Evidências Empíricas}

Camargos e Barbosa (2006) desenvolveram um estudo de eventos para analisar o anúncio de fusões e aquisições das empresas listadas na Bovespa entre julho de 1994 e julho de 2002. Com uma amostra composta por 55 eventos, a janela de estimação utilizada pelos autores foi composta pelos 90 dias que antecederam ao início da janela do evento, que foi constituída por 20 dias antes e após ao evento em questão. Para a estimação dos retornos esperados os autores empregaram o Modelo de Mercado, adaptado pelo procedimento trade-to-trade, de modo em que os dias sem negociação eram desprezados para o cálculo dos retornos. Como principal resultado, cabe destacar que os autores encontraram que houve uma reação positiva, com os preços se ajustando de maneira lenta, sendo um indício de que o mercado se comportou de maneira ineficiente.

Nardy, Famá, Guevara e Mussa, (2015) estudaram se há a ocorrência de retornos anormais no evento do anúncio da incorporação da ação na carteira do Ibovespa. Para tanto é utilizada uma amostra de 43 ativos que ingressaram no índice entre 2004 e 2013. Os autores estimaram os retornos esperados por meio do modelo de mercado, em uma janela de 120 dias, e utilizaram uma janela de evento de 30 dias, para verificar a presença de retornos anormais. Dentre os resultados atestados pelos autores, destaca-se que foi verificada a presença de retornos anormais positivos e significativos nas datas de 3 e 2 dias anteriores ao ingresso dos ativos no lbovespa.

Camargos e Barbosa (2015) estudaram o efeito do anúncio de fusões e aquisições ocorridas entre 1996 e 2004, com uma amostra de 61 ações preferenciais e 27 ações ordinárias. Os autores empregaram o modelo de mercado para estimar os retornos esperados, e utilizaram uma janela de 5 dias em torno da data do evento para observar a presença de retornos anormais. Em relação ao comportamento do mercado, os autores destacam que não houve a presença de retornos anormais estatisticamente diferentes de zero, com isso diz que o mercado se comportou de maneira eficiente para o evento em questão.

Bergmann et al. (2015) e Romano e Almeida (2015), tal como Camargos e Barbosa (2006; 2015) estudaram os impactos de processos de fusões e aquisições no mercado acionário brasileiro. Bergmann et al. (2015) tem como amostra as fusões e aquisições ocorridas entre empresas do setor bancário entre 1994 e 2011, ao passo que Romano e Almeida (2015) estudaram uma série de eventos ocorridos durante o processo de fusão entre a Sadia e Perdigão, anunciada em 2009. Os resultados destes estudos apontam em direções opostas, Bergmann et al. (2015) não encontraram impactos significativos na geração de valor dos bancos envolvidos no processo, já Romano e Almeida (2015) constataram a presença de retornos positivos e estatisticamente significativos, sendo os valores maiores para a empresa adquirida, além disso, estes autores verificaram existirem retornos positivos também para as empresas rivais. 
Em estudo similar ao desenvolvido por Nardy et al. (2015), Silva e Callado (2017) empregaram a metodologia de estudo de eventos para verificar os impactos nos preços, quando da inclusão de uma ação no Índice de Sustentabilidade Empresarial. Para uma amostra composta por 15 ações, os autores, tal como em Camargos e Barbosa (2015), empregaram o modelo de mercado, com uma janela de evento de 5 dias. Silva e Callado (2017) apontaram que não foram encontrados retornos anormais significativos, tendo o mercado se comportado de maneira eficiente para esse evento.

Com intuito de demonstrar as mais variadas formas de utilização dos métodos de estudos de eventos, o quadro 1 apresenta alguns estudos internacionais sobre o tema, como forma de ilustrar a importância dessa metodologia e os principais resultados encontrados em pesquisas desenvolvidas em diferentes mercados.

\begin{tabular}{|c|c|c|}
\hline Referência & Proposta e Amostra & Síntese dos Resultados \\
\hline $\begin{array}{c}\text { Anand e Singh } \\
\qquad(2008)\end{array}$ & $\begin{array}{l}\text { Analisaram, por meio de um } \\
\text { estudo de evento, os efeitos nos } \\
\text { retornos dos acionistas em } \\
\text { cinco fusões no setor bancário } \\
\text { indiano. }\end{array}$ & $\begin{array}{l}\text { Os resultados apontaram a presença de retornos } \\
\text { anormais positivos significativos para os anúncios } \\
\text { analisados, sendo esse efeito tanto para os } \\
\text { compradores quanto para os bancos-alvo. }\end{array}$ \\
\hline Staikouras (2009) & $\begin{array}{l}\text { Examina os efeitos sobre as } \\
\text { ações em decorrência de } \\
\text { alianças entre bancos e } \\
\text { seguradoras. Usa uma amostra } \\
\text { de } 51 \text { eventos, no período de } \\
1990 \text { a } 2006 .\end{array}$ & $\begin{array}{l}\text { Os resultados revelam retornos anormais } \\
\text { significativos quando do evento. Foi constatado } \\
\text { também que os bancos lograram ganhos positivos } \\
\text { significativos, enquanto as seguradoras obtiveram } \\
\text { perdas significativas. }\end{array}$ \\
\hline $\begin{array}{l}\text { Hasan, Schmiedel e } \\
\text { Song (2012) }\end{array}$ & $\begin{array}{l}\text { Avalia os efeitos de Fusões e } \\
\text { Aquisições (F\&A) e alianças na } \\
\text { criação de valor dos acionistas. } \\
\text { Foi utiliza uma amostra com } 111 \\
\text { eventos, em } 15 \text { bolsas de } \\
\text { valores, no período de 2000- } \\
2008 \text {. }\end{array}$ & $\begin{array}{l}\text { O estudo indica uma presença de reações positivas } \\
\text { nos preços das ações a } F \& A \text { e alianças. Aponta-se } \\
\text { que as fusões e aquisições criam mais valor do que } \\
\text { as alianças. Nas alianças, as joint-ventures geram } \\
\text { mais valor do que as alianças não-patrimoniais. }\end{array}$ \\
\hline $\begin{array}{r}\text { Chen, King } \\
(201\end{array}$ & $\begin{array}{l}\text { Investigam o impacto do } \\
\text { anúncio de joint-ventures ou } \\
\text { alianças estratégicas no } \\
\text { mercado de bonds. A amostra é } \\
\text { constituída por } 2.964 \text { anúncios, } \\
\text { ocorridos entre } 1985 \text { e } 2011 .\end{array}$ & $\begin{array}{l}\text { Os autores encontraram uma reação positiva e } \\
\text { significativa no valor tanto das ações, quanto dos } \\
\text { bonds das empresas. A evidências apontam que } \\
\text { não houve transferência de renda entre os } \\
\text { acionistas e os detentores dos bonds. Sendo esse } \\
\text { efeito atribuído principalmente à expectativa de } \\
\text { aumento dos lucros. }\end{array}$ \\
\hline Selcı & $\begin{array}{l}\text { Investiga a criação de riqueza } \\
\text { para os acionistas em } 67 \\
\text { processos de F\&A ocorridos } \\
\text { entre } 2000 \text { e } 2014 \text {, no mercado } \\
\text { turco. }\end{array}$ & $\begin{array}{l}\text { Os resultados mostraram a presença de retornos } \\
\text { anormais acumulados positivos e significativos } \\
\text { para as empresas-alvo. Os autores apontam que a } \\
\text { presença de retornos anormais antes mesmo da } \\
\text { data do evento é um indicio do vazamento de } \\
\text { informações. }\end{array}$ \\
\hline $\begin{array}{l}\text { Mager e Fackler } \\
\qquad(2017)\end{array}$ & $\begin{array}{l}\text { Estudam a performance das } \\
\text { ações de } 338 \text { empresas } \\
\text { adquirentes em processos de } \\
\text { F\&A, ocorridos na Alemanha } \\
\text { entre } 1981 \text { e } 2010 .\end{array}$ & $\begin{array}{l}\text { Para a amostra completa dos dados não foram } \\
\text { encontrados retornos anormais significativos. } \\
\text { Quando os eventos foram divididos em } \\
\text { subamostras, observou-se retornos positivos } \\
\text { anormais, para as F\&A ocorridas entre } 1981 \text { e } 1990 .\end{array}$ \\
\hline
\end{tabular}

\section{Quadro 1. Estudos de Eventos}

Fonte: Elaborado pelos autores (2019)

O cenário apresentado com os estudos empíricos, demonstra que a metodologia dos estudos de eventos é amplamente empregada na literatura, para se testar a reação dos mercados à diferentes eventos. Destaca-se que, entre os estudos mencionados não há uma hegemonia entre os resultados, tendo estudos que apontam para um comportamento eficiente dos mercados e outros que verificam ineficiências nas reações 
dos preços quando do evento, tal controvérsia entre os resultados desses estudos foi verificada tanto no contexto nacional, quanto das pesquisas desenvolvidas em outros mercados, apresentadas no quadro 1.

\subsection{Joint-venture}

Pitofsky (1969) descreve o termo joint-venture a partir das situações em que duas ou mais empresas independentes unem forças em busca de um objetivo comum. Na mesma direção, Child e Faulkner (1998) centram sua definição na cooperação existente entre as empresas, sendo este tipo de relação um mecanismo organizacional empregado na busca por objetivos comuns e ganhos mútuos. A American Bar Association (2006) descreve a joint-venture a partir de uma relação contratual entre duas ou mais empresas, que pode culminar na criação de uma nova empresa, com sua propriedade dividida entre as empresas criadoras, ou restringir-se apenas ao compartilhamento de algum ativo específico.

A relação estabelecida entre as empresas no ato de criação de uma joint-venture pode ser definitiva ou não, com casos inclusive em que desde o momento de sua criação, já é determinado o prazo em que essa relação irá vigorar. Assim, o estabelecimento de uma joint-venture não faz com que as empresas parceiras percam sua personalidade jurídica, nem mesmo seu caráter de competidoras, quando a relação se estabelece entre concorrentes.

Johnson e Houston (2000) apontam que uma joint-venture gera ganhos para as empresas devido as sinergias existentes entre seus negócios. Outras motivações apontadas pelos autores para que as empresas estabeleçam e mantenham esse tipo de relação são diversas, mas destacam-se a necessidade de investimento em ativos específicos, a existência de elevados custos de transação e o compartilhamento de riscos.

Kogut (1988) menciona o fato de que as empresas podem estabelecer essa relação de maneira estratégica para elevar seu poder de mercado. Devido às preocupações em relação ao estabelecimento de uma nova joint-venture e a elevação do poder de mercado, Avellar, Teixeira e Paula (2012) estudam a política antitruste brasileira para os casos de novas joint-ventures. Já D'Agostini, Guerra, Sartor e Tondolo (2014) estudam a formação de joint-ventures com ênfase na relação e nos aspectos estratégicos que são estabelecidos entre os sócios.

Dahab, Guimarães e Dantas (1993) argumentam que esse tipo de relação por vezes é estabelecido como uma ferramenta para a transferência tecnológica entre as empresas. Lopes (2004) apresenta também a utilização de joint-ventures internacionais como uma estratégia para a entrada em um novo mercado. Desse modo, em uma estratégia de internacionalização a multinacional normalmente detém a tecnologia, enquanto a empresa atuante no mercado interno possui um know-how técnico necessário para adequar a tecnologia ao novo contexto.

Com o exposto anteriormente, fica evidenciado que a formação de uma nova joint-venture ocorre quando há a possibilidade de gerar benefícios de alguma forma para as empresas sócias. Sendo assim, esperase que ao criar uma joint-venture os lucros esperados de uma empresa sejam revistos, com um impacto positivo advindo dessa nova relação, com isso o resultado esperado de um anúncio desse tipo é a elevação dos preços das ações das empresas participantes.

McConnell e Nantell (1985) realizam um estudo de evento no qual são analisadas 210 empresas, listadas na American Stock Exchange (ASE) e na New York Stock Exchange (NYSE), envolvendo 136 jointventures, no período de 1972-1979. Seus resultados apontam para a existência de um retorno positivo e estatisticamente significante nas ações das empresas que anunciam uma joint-venture.

Johnson e Houston (2000) analisam os efeitos criados com o anúncio de 119 joint-ventures americanas, no período de 1991-1995. Seus resultados apontam para a existência de ganhos para ambas os sócios no caso de uma joint-venture em relações horizontais, já para os casos de joint-ventures verticais, os resultados apontam ganhos apenas para os fornecedores, sendo as empresas compradoras beneficiadas de outras formas.

Em estudo sobre os efeitos do anúncio de uma joint-venture internacional, Liu, Aston e Acquaye (2014) analisam 394 casos entre 2005 e 2010. É apontado no trabalho que a literatura mostra a existência de retornos em excesso estatisticamente significantes para joint-ventures nacionais, já quando se trata de joint- 
venturesinternacionais os resultados são inconclusivos. Como resultado do estudo foi constatado que as jointventures internacionais criam valor, mas que esse efeito não é duradouro na avaliação do mercado.

\section{Metodologia}

Em relação ao objetivo, essa pesquisa pode ser classificada como descritiva, conforme exposto por Gil (2008), esse tipo de pesquisa visa descrever as características de um fenômeno, com o estabelecimento de relação entre variáveis. A pesquisa pode ser classificada ainda, conforme exposto por Mazzotti e Gewandsznajder (2001), como quantitativa, uma vez que, propõe um método investigativo com intuito de realizar um exame detalhado dos conceitos para maior entendimento e precisão do evento analisado.

\subsection{Estudo de eventos}

Neste artigo será utilizado o método proposto por Campbell et al. (1997) para a verificação do impacto do evento nos preços das ações. $O$ método pode ser dividido em sete etapas, representadas de maneira simplificada na Figura 1, de forma a facilitar a compreensão das etapas realizadas para o desenvolvimento desta pesquisa.

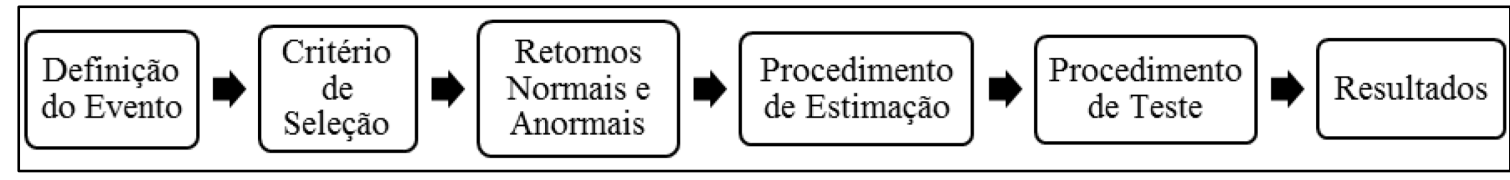

Figura 1. Etapas do Estudo de Eventos

Fonte: Adaptado de Camargos e Barbosa (2006)

\subsubsection{Definição do evento}

O evento analisado foi o anúncio da criação de uma joint-venture em companhias brasileiras de capital aberto. Como data do evento foi considerado o dia da divulgação ao mercado da criação da jointventure. Tal como em Camargos e Barbosa (2015), optou-se por utilizar uma janela de evento de 5 dias em torno da data zero, com isso foram observados 5 dias de negociações antes e depois, para a observação do fenômeno, haja vista, que períodos maiores poderiam incorporar outros eventos corporativos diferentes do analisado.

\subsubsection{Critério de seleção da amostra}

Incialmente foi feito um levantamento das empresas brasileiras de capital aberto que anunciaram e concluíram acordos de criação de joint-venture, no período de 24/05/2012 a 24/05/2017. Nesse sentido, a amostra do estudo é não probabilística e intencional, resultando em uma amostra inicial de 12 casos de criação de novas joint-ventures envolvendo empresas brasileiras com ações negociadas em bolsa de valores, sendo considerada para o estudo apenas a ação de maior liquidez de cada empresa. Ressalta-se que o levantamento das empresas e as datas de anúncio da criação das joint-ventures, bem como as cotações diárias das ações dessas empresas, devidamente ajustadas para pagamento de dividendos, bonificações, splits e implits, foram obtidas por meio de plataforma de informações financeiras Bloomberg.

As ações que compõem a amostra do estudo, as joint-ventures e as respectivas datas de anúncio da criação das joint-ventures são apresentadas na Tabela 1. Ressalta-se que, a EMBR3 e a CSAN3 aparecem em duas oportunidades, em razão dessas empresas apresentarem o anúncio de mais de uma joint-venture no 
período amostral. Destaca-se também que, devido à ausência de informações sobre o nome de algumas das joint-ventures na Bloomberg, foi indicado na Tabela 1 apenas o código informado na plataforma.

\begin{tabular}{clc}
\hline Empresa & \multicolumn{1}{c}{ Joint-Venture } & \multicolumn{1}{c}{ Anúncio } \\
\hline VALE5 & Aliança Geração Energia S.A & $20 / 12 / 2013$ \\
BRFS3 & Joint Venture- 6112012 & $06 / 11 / 2012$ \\
TELB3 & Visiona Tecnologia Espacial S.A & $29 / 05 / 2012$ \\
EMBR3 & Visiona Tecnologia Espacial S.A & $29 / 05 / 2012$ \\
EMBR3 & Joint Venture- 12062012 & $12 / 06 / 2012$ \\
ITUB4 & Banco Itau Consignado S.A & $10 / 07 / 2012$ \\
PFRM3 & Profarma Specialty & $24 / 03 / 2014$ \\
ODPV3 & Brasildental Operadora de Plano Odontológicos S.A. & $11 / 06 / 2013$ \\
BBAS3 & Brasildental Operadora de Plano Odontológicos S.A. & $11 / 06 / 2013$ \\
CSNA3 & Joint Venture- 24112014 & $24 / 11 / 2014$ \\
CSAN3 & Novvi LLc & $19 / 07 / 2016$ \\
GGBR4 & Gerdau Summit Aços Fundidos de Forjados S.A & $28 / 01 / 2016$ \\
\hline
\end{tabular}

Tabela 1. Amostra do Estudo

Fonte: Elaborada pelos autores (2019)

\subsubsection{Retornos normais e anormais}

Diante do objetivo da pesquisa, apresenta-se na equação 1, o modelo sugerido por Campbell et al. (1997), empregado nesse estudo para o cálculo do retorno anormal das ações das empresas acionistas nas joint-ventures durante a janela de evento.

$$
R A_{i t}=R_{i t}-E\left(R_{i t}\right)
$$

Em que, RAit é o retorno anormal da empresa i no período t; Rit é o retorno observado; $E$ (Rit) é o retorno esperado, estimado por meio do modelo descrito no tópico 3.1.4; e os subscritos $i$ e t referem-se à ação em questão e ao tempo do evento, respectivamente. Desse modo, o RAit das ações, é obtido pela diferença entre o retorno observado para a ação e o retorno esperado sem a ocorrência do evento.

Uma vez calculados os retornos anormais, estes foram acumulados em cada um dos dias da janela de evento, conforme técnica do Retorno Anormal Acumulado (CAR) proposta por Campbell et al. (1997), descrita na equação (2).

$$
C A R=\frac{1}{n} \sum_{i=1}^{n} R A_{i t}
$$

Em que, CAR é o retorno anormal acumulado até uma data específica na janela do evento, $\mathrm{n}$ é o número de empresas na amostra, e RA é o retorno anormal da empresa $i$ até a data t em que se deseja calcular o CAR.

\subsubsection{Procedimento de estimação}

A janela de estimaação foi composta pelos 200 dias de negociação anteriores ao início da janela do evento, conforme Figura 2. 


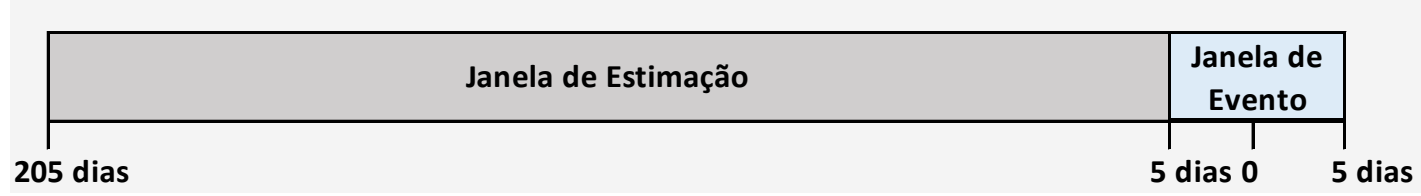

Figura 2. Esquema de Janelas

Fonte: Elaborada pelos autores (2019)

O retorno esperado foi obtido por meio de uma regressão linear, tendo como variável dependente os retornos observados para cada ativo durante a janela de estimação, e como variável independente os retornos para a proxy de mercado, sendo considerado o índice IBOVESPA neste estudo. Para verificar a existência de problemas com os modelos estimados foram realizados os testes de Breusch-Pagan, para verificar a presença de heteroscedasticidade e o teste de Durbin-Watson na verificação da presença de autocorrelação nos modelos. Diante da verificação de autocorrelação nos modelos de algumas ações, para essas, tal como em Bergmann et al. (2015), utilizou-se o método de Newey e West (1987) para correção dos erros-padrão, como número de lags para essa estimação, considerou-se a parte inteira de $\mathrm{n} 1 / 4$, em que $n$ representa o número de observações da amostra, conforme sugerido por Wooldridge (2011).

\subsubsection{Procedimento de teste}

Dado o objetivo da pesquisa, foi aplicado, incialmente, o teste de Shapiro-Wilk para verificar a presença da distribuição normal na amostra dos retornos anormais das ações em cada dia da janela de evento, sendo este teste escolhido por se adequar ao tamanho da amostra do estudo. Posteriormente foi empregado o teste T de student em caso de normalidade da amostra, ou o teste de Wilcoxon, em caso de não normalidade da amostra, em procedimento similar ao empregado por Nardy et al. (2015).

A hipótese nula de retornos anormais acumulados a ser testada é:

$\mathrm{HO}: \mu=0$

No que se refere aos testes, foi considerado o nível de significância de $5 \%$ nas análises. Foi então analisada a significância estatística dos retornos anormais acumulados nos dias da janela do evento, visando identificar se são diferentes de zero. Para a não rejeição da hipótese nula os resultados devem demonstrar que os retornos anormais acumulados são, estatisticamente, insignificantes e iguais à zero, e, neste caso, o mercado comportou-se de maneira eficiente no que se refere à incorporação da informação do anúncio de criação de uma joint-venture. Ressalta-se que a estimação dos modelos, a acumulação dos retornos anormais e os testes foram feitos utilizando programação em linguagem Python.

\section{Resultados}

Esta seção do trabalho dedica-se a apresentação dos resultados dos modelos econométricos, bem como dos testes realizados com o objetivo de verificar se o mercado foi eficiente para o evento em estudo. Além disso, é feita uma discussão dos resultados em relação a outros trabalhos, sendo destacados os pontos de similaridade e de divergência entre este estudo e os estudos prévios.

\subsection{Modelos Econométricos}

A Tabela 2 apresenta um resumo dos resultados dos modelos estimados para o cálculo dos retornos esperados. Para todos os testes realizados neste estudo o nível de significância adotado foi de $5 \%$. Inicialmente, 
em relação ao teste de Breusch-Pagan (BP), ressalta-se que dado o nível de significância adotado, em nenhum caso rejeitou-se a hipótese nula de que a variância dos resíduos é constante, com isso constatou-se que a heteroscedasticidade não é um problema para os dados das ações que compõem a amostra da pesquisa.

Em relação ao teste de Durbin-Watson (DW) para autocorrelação, os limites para a realização do teste foram definidos de acordo com o tamanho da amostra $(n=200)$ e o número de variáveis explicativas consideradas nos modelos $(k=1)$. Os limites adotados foram então de 1,758 para dL e 1,778 para dU. Conforme destacam Gujarati e Porter (2011), se o valor calculado para a estatística d estiver entre dU e 4-dU não se rejeita a hipótese nula de que não há a presença de autocorrelação. Para as ações TELB3, ITUB4, PFRM3 e BBAS3 o valor de d é inferior até mesmo ao valor de dL, o que, conforme destacado por Gujarati e Porter (2011), nos permite rejeitar a hipótese nula, sendo uma evidência da presença de autocorrelação positiva. Conforme já destacado na metodologia, para essas ações os modelos foram estimados novamente, com os erros-padrão sendo corrigidos pelo método de Newey-West.

Por fim, foi analisado o p-valor para o coeficiente Beta estimado para as regressões. Sendo que, os valores considerados na Tabela 2 já estão corrigidos pelo procedimento de Newey-West para as ações que apresentaram autocorrelação. Dado o nível de significância de 5\%, os coeficientes das ações TELB3 e PFRM3 não foram estatisticamente significativos, com isso, para as análises que se seguem neste estudo, os dados referentes a essas ações foram excluídos da amostra.

\begin{tabular}{ccccc}
\hline & Beta & p-valor & p-valor BP & d de DW \\
\hline VALE5 & 1,034162 & $5,64 \mathrm{E}-30^{*}$ & 0,323124 & 2,112994 \\
BRFS3 & 0,556864 & $2,35 \mathrm{E}-11^{*}$ & 0,74758 & 2,048984 \\
TELB3 & $-0,05248$ & 0,740066 & 0,691564 & 1,649647 \\
EMBR3 & 0,881562 & $3,51 \mathrm{E}-24^{*}$ & 0,164923 & 2,152148 \\
EMBR3 & 0,742815 & $8,12 \mathrm{E}-17^{*}$ & 0,350626 & 2,225076 \\
ITUB4 & 0,909812 & $1,69 \mathrm{E}-38^{*}$ & 0,923725 & 1,632045 \\
PFRM3 & $-0,20617$ & $0,078208^{* * *}$ & 0,433107 & 1,538043 \\
ODPV3 & 0,415525 & $8,3 \mathrm{E}-05^{*}$ & 0,343791 & 2,30867 \\
BBAS3 & 0,818563 & $8,03 \mathrm{E}-13^{*}$ & 0,096067 & 1,730634 \\
CSNA3 & 0,931795 & $4,62 \mathrm{E}-15^{*}$ & 0,399342 & 1,928517 \\
CSAN3 & 0,721473 & $4,01 \mathrm{E}-18^{*}$ & 0,185922 & 2,280152 \\
GGBR4 & 1,145352 & $2,55 \mathrm{E}-14^{*}$ & 0,301300 & 1,885948 \\
\hline
\end{tabular}

Nota. ${ }^{*}$ valores significativos a $1 \% ;{ }^{* *}$ valores significativos a $5 \% ;{ }^{* * *}$ valores significativos a $10 \%$.

\section{Tabela 2. Resultados das Regressões para os Retornos Esperados}

Fonte: Elaborada pelos autores (2019)

\subsection{Retornos anormais}

Na Tabela 3 são apresentados os retornos anormais (AR) e o retorno anormal acumulado (CAR) entre todas as ações da amostra durante a janela de evento. Observa-se um comportamento de queda nos retornos anormais durante a janela do evento, sendo que apenas no dia que antecede o anúncio da criação da jointventure, no dia do próprio anúncio e no quinto dia após o anúncio foram verificados retornos anormais positivos. Isso reflete-se em um CAR altamente negativo durante toda a janela de evento, findando em 4,17743\% acumulados nos 11 dias analisados. A Figura 3 auxilia na compreensão do comportamento do CAR durante a janela de evento, sendo possível visualizar graficamente que, apesar das datas em que há um AR positivo, sua magnitude não é o suficiente para tornar o CAR positivo. 


\begin{tabular}{ccc}
\hline Dia & AR & CAR \\
\hline-5 & $-0,88432$ & $-0,88432$ \\
-4 & $-0,60472$ & $-1,48903$ \\
-3 & $-0,43282$ & $-1,92185$ \\
-2 & $-1,83004$ & $-3,75189$ \\
-1 & 0,711656 & $-3,04024$ \\
0 & 0,294872 & $-2,74537$ \\
1 & $-0,61902$ & $-3,36438$ \\
2 & $-0,48262$ & $-3,84701$ \\
3 & $-0,10175$ & $-3,94875$ \\
4 & $-0,59835$ & $-4,5471$ \\
5 & 0,369669 & $-4,17743$ \\
\hline
\end{tabular}

Tabela 3. Retornos Anormais na Janela do Evento

Fonte: Elaborada pelos autores (2019)

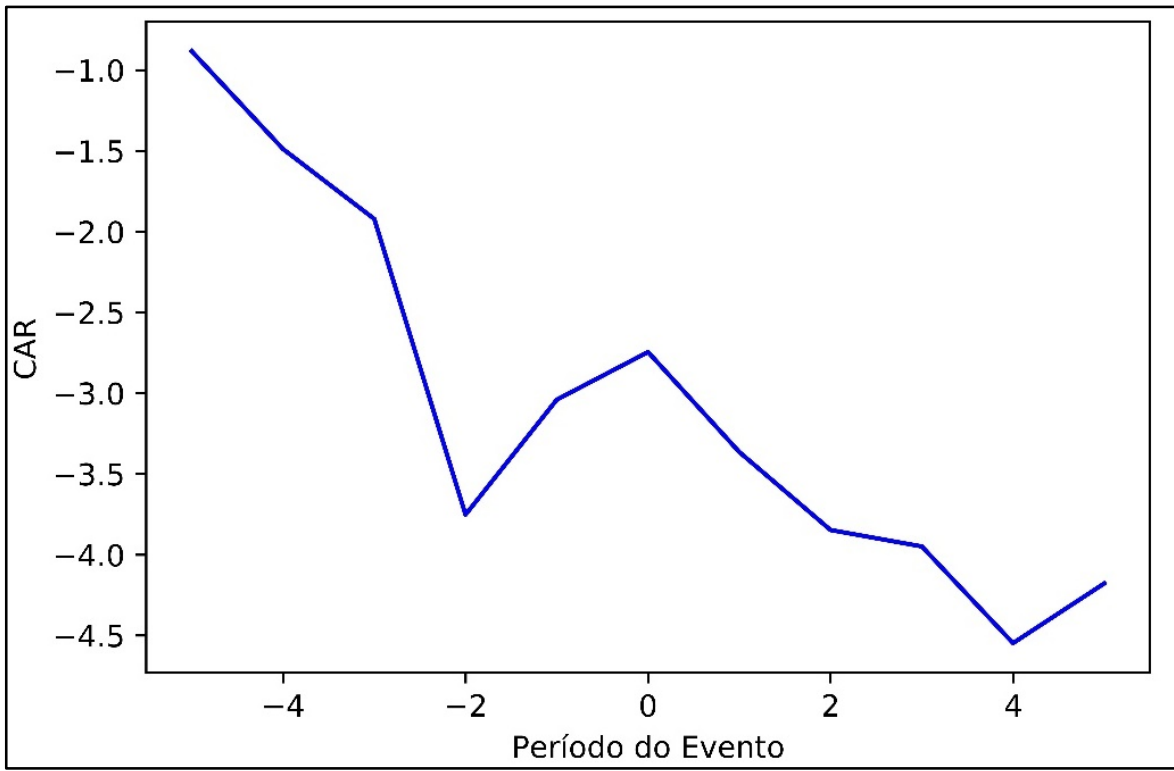

Figura 3. Retorno Anormal Acumulado

Fonte: Elaborada pelos autores (2019)

\subsection{Teste de hipótese para os retornos}

Por fim, na Tabela 4 são evidenciados os resultados para o teste de normalidade de Shapiro-Wilk e o teste para a comparação das médias dos retornos anormais (AR) das ações em cada dia da janela de evento. Ressalta-se que o teste para a normalidade possui a hipótese nula de que os dados seguem uma distribuição normal, a coluna "normal" na tabela 4 apresenta o p-valor resultante desse teste para cada dia de evento, na análise desses valores é possível observar que apenas no dia -3 os dados não seguiram uma distribuição normal, para essa data, portanto foi empregado o teste de Wilcoxon, no restante dos dias da janela de evento o teste $\mathrm{T}$ foi utilizado para verificar se a média dos retornos anormais foi estatisticamente igual a zero. 


\begin{tabular}{ccl}
\hline Dia & normal & \multicolumn{1}{c}{ p-valor } \\
\hline-5 & 0,995968 & $0,077227^{* * *}$ \\
-4 & 0,957523 & $0,0795^{* * *}$ \\
-3 & $0,001628^{*}$ & 0,721277 \\
-2 & 0,357632 & $0,016034^{* *}$ \\
-1 & 0,742019 & $0,004492^{*}$ \\
0 & 0,683643 & 0,638343 \\
1 & 0,158217 & 0,160276 \\
2 & 0,12184 & 0,628694 \\
3 & 0,568093 & 0,898291 \\
4 & 0,126419 & 0,319022 \\
5 & 0,103408 & 0,693589 \\
\hline a $\%{ }^{* *}$ valores significativos a $5 \% ;{ }^{* * *}$ valores significativos a $10 \%$.
\end{tabular}

Tabela 4. Estatísticas dos Retornos na Janela do Evento

Fonte: Elaborada pelos autores (2019)

Na coluna p-valor da Tabela 4 são apresentados os resultados do teste para as médias, ressalta-se que tanto o teste T, quanto o teste de Wilcoxon possuem a mesma hipótese nula, de que a média da amostra é igual a zero, o que implica que se essa hipótese não for rejeitada, o mercado se comportou de maneira eficiente. Na análise do AR para as ações da amostra é possível observar que os retornos anormais foram estatisticamente diferentes de zero apenas nos dois dias que antecederam ao evento, quando considerado $o$ nível de significância estatística de 5\%. A fim de observar também se o resultado para o CAR na janela de evento foi estatisticamente diferente de zero, realizou-se procedimento similar para o retorno anormal acumulado por todas as ações no último dia da janela de evento. $O \mathrm{p}$-valor para o teste de normalidade para o CAR foi de 0,5762 , foi então empregado o teste paramétrico que retornou um p-valor de 0,1696 , que não permite rejeitar a hipótese nula de que o CAR para as ações da amostra foi diferente de zero durante a janela de evento.

\subsection{Análise dos resultados}

Os resultados expostos anteriormente evidenciam que, apenas nos dois dias que antecederam o anúncio da criação da joint-venture foram verificados retornos anormais estatisticamente significativos. Se esse resultado for comparado com as evidências da Tabela 3, vê-se que o AR nessas datas sofre uma mudança de direção, sendo que o valor negativo do dia -2 é superior ao retorno positivo do dia anterior do evento.

O fato anteriormente exposto pode ser considerado uma evidência de que houve o uso de informação privilegiada para negociação no mercado, sendo observada uma reação negativa e abrupta, com uma correção no dia seguinte. Em linha com Bergmann et al. (2015), este resultado é um indicativo da importância de se analisar o comportamento dos preços nos dias que antecedem a ocorrência do evento. No caso deste estudo as evidências apontam para a existência de negociações explorando a informação antes que ela fosse divulgada ao mercado, e também para uma interpretação negativa dos efeitos da criação de uma nova jointventure para os seus acionistas, dado o comportamento dos preços nos dias que antecederam ao evento.

Nos demais dias da janela de evento e no CAR acumulado ao longo dos 11 dias de evento não foram observados retornos anormais estatisticamente significativos, com isso nessas datas o mercado teria se comportado de maneira eficiente, em seu nível semiforte. Com relação ao retorno nas datas -2 e - 1 , que foram estatisticamente significativos, podendo ser essa análise estendida a toda a janela de evento, mesmo para os dias que não foram significantes, viu-se que a tendência nos ARs é fortemente negativa, sendo esse resultado contraditório com o exposto por McConnell e Nantell (1985) que atestam para a presença de retornos anormais 
positivos para esse tipo de evento, esse resultado também está em desacordo com os achados de Liu et al. (2014).

Em suma, os resultados deste estudo estão em desacordo com os achados de McConnell e Nantell (1985), Johnson e Houston (2000), Liu et al. (2014) e de Chen et al. (2015), que encontram retornos positivos quando da criação de novas joint-ventures. Desse modo, em contraste com a literatura internacional, no mercado brasileiro a divulgação da criação de uma nova joint-venture é vista como um evento negativo. Este resultado também está em desacordo com estudos que analisam eventos de fusões e aquisições no mercado brasileiro, como é o caso de Camargos e Barbosa (2006, 2015), Bergmann et al. (2015) e Romano e Almeida (2015).

A despeito da literatura que aponta para os aspectos positivos para os acionistas da joint-venture como é o caso dos trabalhos de Kogut (1988), Dahab, Guimarães e Dantas (1993) e Johnson e Houston (2000), existem evidências que apontam para o elevado número fracasso neste tipo de relação. Park e Russo (1996) investigam os motivadores para o insucesso de joint-ventures, no que tange a interpretação negativa da criação de uma joint-venture, esses autores argumentam que em muitos casos a competição entre os acionistas torna-se maior que a cooperação que motivou o estabelecimento da cooperação, culminando em uma relação fracassada e em muitos casos de parasitismo e disputa pelo poder.

Park e Russo (1996) demonstram que a maior parte dos fracassos de joint-ventures ocorre no médio prazo após o estabelecimento da relação, sendo a falha nas estruturas de governanças internas da nova empresa e a falta de uma governança interorganizacional um dos fatores que levam ao fracasso da relação. Desse modo, a precificação negativa, que ocorre quando do evento do anúncio da criação de uma nova jointventure, pode ser interpretada como uma antecipação do mercado para os riscos deste tipo de relação fracassar e impactar negativamente as empresas acionistas.

Em relação ao comportamento do mercado em si, ressalta-se novamente o fato de que apenas nos dois dias que antecederam o evento foram observados retornos anormais significativos, nos demais dias da janela de evento o mercado se comportou de maneira eficiente. Tais resultados estão mais próximos do que foi encontrado nos trabalhos de Bergmann et al. (2015), Camargos e Barbosa (2015) e Silva e Callado (2017), que apontam para um comportamento eficiente do mercado brasileiro.

\section{Considerações Finais}

O presente trabalho foi realizado com o objetivo de analisar a eficiência de mercado, das empresas brasileiras, no evento do anúncio de criação de uma joint-venture. Para tanto, foram levantados na plataforma de informações financeiras da Bloomberg os anúncios de novas joint-ventures envolvendo empresas brasileiras no período de 24/05/2012 a 24/05/2017. De posse das datas dos anúncios, das empresas acionistas e das cotações das acionistas que possuíam ações negociadas em bolsa, foi empregada a metodologia de estudo de evento para se fazer a análise proposta no objetivo do trabalho.

$\mathrm{Na}$ condução do estudo de evento foram seguidos os passos descritos por Campbell et al. (1997), sendo adotada uma janela de estimação de 200 dias e uma janela de evento de 5 dias em torno da data zero. Os retornos esperados foram estimados pelo modelo de mercado, sendo esse corrigido pelo procedimento de Newey-West quando foi atestada a presença de autocorrelação. Após estimados os modelos para o cálculo do retorno esperado, foram então calculados os retornos anormais e os retornos anormais acumulados durante a janela de evento. Por fim, foi realizado o teste de Shapiro-Wilk para atestar a presença de normalidade para os ARs e para o CAR, e então foi aplicado o teste mais adequado, teste T de student ou teste de Wilcoxon, para verificar se o resultado foi estatisticamente significativo.

Os resultados do trabalho evidenciaram uma forte tendência de queda nos ARs ao longo da janela de evento, sendo que apenas nos dias -1, 0 e 5 seu valor foi positivo, consequentemente o CAR da janela de evento foi fortemente negativo, atingindo um valor de $-4,17 \%$ no final da janela de evento. Tais valores negativos indicam que o anúncio de uma joint-venture, foi visto como algo negativo pelo mercado, corroborando com Park e Russo (1996), que ressaltam o grande número de casos de fracasso nesse tipo de relação, muito em decorrência da competição predominar sobre a cooperação entre as empresas. Sobre a significância desses 
resultados ressalta-se que apenas nos dias -2 e -1 o AR foi estatisticamente significativo, mostrando que nas demais datas o resultado não foi estatisticamente diferente de zero, quando testada a significância do CAR da janela de evento esse também não se mostrou estatisticamente significativo.

Finalmente, ressalta-se que o trabalho cumpriu seu objetivo ao verificar que o mercado se comportou de maneira eficiente na maior parte dos dias da janela de evento, sendo que apenas nos dois dias que antecederam o anúncio não houve um comportamento eficiente, indicando a possibilidade do vazamento de informações sobre a criação da joint-venture. Verificou-se também que a informação do estabelecimento dessa relação foi vista como algo negativo pelo mercado. Esse resultado pode ser útil principalmente para os gestores de empresas que avaliam a possibilidade de estabelecer uma nova joint-venture, mostrando os impactos que essa decisão pode ocasionar nas ações de sua empresa. Como sugestão para pesquisas futuras sugere-se a incorporação de outros mercados na amostra para a comparação dos resultados, além disso destaca-se a possibilidade da utilização de outros modelos, além do modelo de mercado, e de outros tamanhos de janelas, para atestar a consistência dos resultados.

\section{REFERÊNCIAS}

American Bar Association - ABA. (2006). Joint-ventures: analysis of collaborations among competitors (2. ed.). Chicago: American Bar Association.

Anand, M., \& Singh, J. (2008). Impact of merger announcements on shareholders' wealth: evidence from indian private sector banks. Vikalpa: Journal for Decision Makers, 33(1), 35-54.

Avellar, A. P., Teixeira, H. A., \& Paula, G. M. (2012). Joint-ventures e a política antitruste brasileira. Revista de Economia Contemporânea, 16(3), 463-486.

Bergmann, D. R., Savoia, J. R. F., Souza, B. M., \& Mariz, F. (2015). Avaliação dos Processos de Fusões e Aquisições no setor Bancário Brasileiro por meio de Estudo de Eventos. Revista Brasileira de Gestão de Negócios, 17(56), 1105-1115.

Camargos, M. A., \& Barbosa, F. V. (2003). Teoria e evidência da eficiência informacional do mercado de capitais brasileiro. Caderno de Pesquisas em Administração, 10(1), 41-55.

Camargos, M. A., \& Barbosa, F. V. (2006). Eficiência informacional do mercado de capitais brasileiro pós-Plano Real: um estudo de eventos dos anúncios de fusões e aquisições. Revista de Administração, 41(1), 4358.

Camargos, M. A., \& Barbosa, F. V. (2015). Eficiência informacional do mercado de capitais brasileiro em anúncios de fusões e aquisições. Production, 25(3), 571-584.

Campbell, J. Y., Lo, A. W., \& MacKinlay, A. C. (1997). The econometrics of financial markets (2a ed.). New Jersey: Princeton University Press.

Chen, J., King, T. H. D., \& Wen, M. M. (2015). Do joint ventures and strategic alliances create value for bondholders? Journal of Banking \& Finance, 58, 247-267.

Child, J., \& Faulkner, D. (1998). Strategies of cooperation: managing alliances, networks and joint ventures. New York: Oxford University Press.

D’Agostini, M., Guerra, R. M. A., Sartor, R. M., \& Tondolo, V. A. G. (2014). Aspectos estratégicos e relacionais das alianças do tipo joint-venture: o caso de dois fabricantes da indústria automobilística. Revista Eletrônica de Ciência Administrativa, 13(2), 267-285.

Dahab, S. S., Guimarães, F., \& Dantas, J. R. (1993). Transferência tecnológica e joint-ventures no Brasil. Organização \& Sociedade, 1(1), 72-96.

Damodaran, A. (2001). Corporate finance: and practice. New York: John Wiley \& Sons.

Fama, E. F. (1970). Efficient capital markets: a review of theory and empirical work. The Journal of Finance, 25(2), 383-417.

Fama, E. F. (1991). Efficient capital markets: II. The Journal of Finance, 46(5), 1575-1617.

Fé Júnior, A. L. D., Nakao, S. H., \& Ribeiro, M. S. (2015). Reações do mercado acionário na primeira divulgação financeira em IFRS do setor bancário brasileiro: um estudo de evento. CONTEXTUS - Revista Contemporânea de Economia e Gestão, 13(3), 81-110.

French, K. R., \& Roll, R. (1986). Stock returns variances: the arrival of information and the reaction of traders. Journal of Financial Economics, 17(1), 5-26.

Gabriel, F. S., Ribeiro, R. B., \& Ribeiro, K. C. S. (2013). Hipóteses de mercado eficiente: um estudo de eventos a partir da redução do IPI. Revista de Gestão, Finanças e Contabilidade, 3(1), 36-52.

Gil, A. C. (2008). Métodos e técnicas de pesquisa social(6a ed.). São Paulo: Atlas. 
Hasan, I., Schmiedel, H., \& Song, L. (2012). Growth strategies and value creation: what works best for stock exchanges? The Financial Review, 47(3), 469-499.

Johnson, S. A., \& Houston, M. B. (2000). A Reexamination of the motives and gains in joint-ventures. The Journal of Financial and Quantitative Analysis, 35(1), 67-85.

Kogut, B. (1988). Joint-ventures: theoretical and empirical perspectives. Strategic Management Journal, 9, 319-332.

Liu, J., Aston, J., \& Acquaye, D. (2014). International joint-ventures: do they enhance shareholder value? [Working Paper № 1403]. Regent's Working Papers in Business \& Management, London, UK.

Lopes, F. D. (2004). Perspectivas teóricas contemporâneas nos estudos das organizações: apresentação de um modelo de análise da formação de joint ventures internacionais. Cadernos EBAPE.BR, 2(3).

Mager, F., \& Fackler, M. M. (2017). Mergers and acquisitions in Germany: 1981-2010. Global Finance Journal, 34, 32-42.

Maluf Filho, J. A. (1991). Eficiência do mercado de opções da Bolsa de Valores de São Paulo. Revista de Administração, 26(3), 12-22.

Mazzotti, A. J. A., \& Gewandsznajder, F. (2001). O método nas ciências naturais e sociais: pesquisa quantitativa e qualitativa (3a ed.). São Paulo: Pioneira.

McConnell, J. J., \& Nantell, T. J. (1985). Corporate combinations and common stock returns: the case of joint ventures. The Journal of Finance, 40(2), 519-536.

Nardy, A., Famá, R., Guevara, J. A. H., \& Mussa, A. (2015). Verificação da ocorrência do efeito índice no Ibovespa - 2004-2013. Revista de Administração, 50(2), 153-168.

Newey, W. K., \& West, K. D. (1987). A simple, positive semi-definite, heteroskedasticity and autocorrelation consistent covariance matrix. Econometrica, 55(3), 703-708.

Park, S., \& Russo, M. (1996). When Competition Eclipses Cooperation: An Event History Analysis of Joint Venture Failure. Management Science, 42(6), 875-890.

Perobelli, F. F. C., \& Ness Júnior, W. L. (2000, setembro). Reações do mercado acionário a variações inesperadas nos lucros das empresas: um estudo sobre a eficiência informacional no mercado brasileiro. Anais do Encontro Nacional da Associação Nacional de Pós-Graduação e Pesquisa em Administração, Florianópolis, SC, Brasil, 24.

Pitofsky, R. (1969). Joint-ventures under the antitrust laws: some reflections on the significance of Penn-Olin. Harvard Law Review, 82(5), 1007-1063.

Romano, P. R., \& Almeida, V. S. (2015). Análise dos Efeitos em Mercado de Capitais Decorrentes de Fusões: O Caso BRF S.A.. Revista de Administração Contemporânea, 19(5), 606-625.

Selcuk, E. A. (2015). Do mergers and acquisitions create value for Turkish target firms? An event study analysis. Procedia Economics and Finance, 30, 15-21.

Silva, N. E. F., \& Callado, A. L. C. (2017). Inclusão de empresas no índice de sustentabilidade empresarial: uma análise do desempenho acionário. Revista de Gestão e Contabilidade da UFPI, 4(1), 134-149.

Staikouras, S. K. (2009). An event study analysis of international ventures between banks and insurance firms. International Financial Markets, Institutions and Money, 19(4), 675-691.

Wooldridge, J. M. (2011). Introdução à econometria: uma abordagem moderna. São Paulo: Cengage Learning. 